\title{
Tempo de fornecimento da dieta pré-inicial para frangos de corte com diferentes pesos ao alojamento ${ }^{1}$
}

\author{
Gilson Alexandre Gomes², Lúcio Francelino Araújo², Joel Alberto Prezzi², Davi Savietto², \\ José Roberto Simioni Júnior ${ }^{2}$, Joseliana Valério²
}

\footnotetext{
1 Projeto financiado pela FAPESP (proc. $n^{\circ}$ 05/01746-0).

2 Faculdade de Zootecnia e Engenharia de Alimentos/USP - Departamento de Zootecnia.
}

\begin{abstract}
RESUMO - Avaliaram-se os efeitos do fornecimento da dieta pré-inicial (DPI) e do peso ao alojamento sobre o desempenho e as características de carcaça de frangos de corte. Utilizaram-se 960 frangos machos, linhagem comercial Cobb-500, alojados em 32 boxes, em um delineamento inteiramente casualizado em esquema fatorial $2 \times 4$, composto de dois pesos ao alojamento (42 e $46 \mathrm{~g})$ e quatro períodos de fornecimento da dieta pré-inicial $(0,1$ a 7,1 a 10 e 1 a 14 dias de idade), durante 49 dias. Os programas alimentares compreenderam três ou quatro fases (pré-inicial e/ou inicial; engorda; final) e as dietas fornecidas eram à base de milho e farelo de soja. De modo geral, aves mais pesadas ao alojamento apresentaram, ao longo de todo o período experimental, maior consumo de ração (CR) e ganho de peso (GP). Não foram observadas diferenças significativas na conversão alimentar (CA) dos animais. Considerando todo o período experimental, as aves alimentadas com a dieta pré-inicial durante sete dias apresentaram maior consumo de ração e ganho de peso, porém, menor rendimento de carcaça. O peso ao alojamento determina o desempenho e o oferecimento da dieta préinicial durante sete dias beneficia o desempenho das aves.
\end{abstract}

Palavras-chave: desempenho, nutrição pós-eclosão, peso do pintinho de 1 dia, rendimento de carcaça

\section{Period of feeding a pre-starter diet for broiler chickens with different body weights at housing}

\begin{abstract}
The effects of feeding a pre-starter diet (PSD) and weight at housing on performance and carcass characteristics broilers were evaluated. A total of 960 day-old male chickens, commercial line Cobb-500, was housed in 32 floor pens as a completely randomized design in a $2 \times 4$ factorial arrangement [weight at housing (42 and 46 g) and periods of feeding pre- starter diet ( 0,1 to 7,1 to 10 and 1 to 14 days old) for 49 days. The feeding regimes contained three or four phases (pre-starter and/or starter; grower; finisher diets) and diets were based on corn and soybean meal. Overall, birds with heavier weights at housing showed, throughout the trial period, greater feed consumption and weight gain. No differences were observed for feed conversion of the animals. Considering the whole trial period, birds fed PSD during seven days showed higher feed consumption and weight gain, however, lower carcass yield. Weights at housing determine the performance and feeding pre-starter diet during first seven days is beneficial for the performance of birds.
\end{abstract}

Key Words: carcass yield, 1-d old chick weight, performance, post-hatching nutrition

\section{Introdução}

Nos últimos anos, a nutrição inicial de frangos de corte tem despertado grande interesse de profissionais que atuam na avicultura. Esse interesse crescente é resultado não apenas das adaptações morfo-fisiológicas das aves no período logo após a eclosão, mas também em virtude da evolução da avicultura brasileira.

Há um considerável interesse sobre a precisão de formular uma dieta para as aves com a perspectiva de diminuir a mortalidade inicial e melhorar a uniformidade do lote.
Adicionalmente, o conhecimento das peculiaridades metabólicas e fisiológicas no período pós-eclosão dão subsídios para a otimização do manejo alimentar, permitindo que as aves expressem todo o seu potencial genético.

A seleção para rápido crescimento em frangos de corte resultou em aumento do apetite, do peso vivo e dos rendimentos de cortes e melhora da conversão alimentar, reduzindo assim o número de dias para o abate (Joseph \& Moran Jr., 2005a). Atualmente, a primeira semana de vida da ave corresponde a $22 \%$ do período de criação, ao passo que em 1978 a primeira semana representava apenas $11 \%$. 
Moran Jr. (1985) e Croom et al. (1999) ressaltaram que as razões que justificariam a adoção de uma dieta diferenciada na primeira semana de idade das aves são a anatomia e fisiologia diferenciadas do trato digestório, a dificuldade de digestão de absorção de alguns nutrientes, o grande potencial de desenvolvimento neste período, além da dificuldade de sobrevivência em ambientes frios.

Em virtude do aumento na produção de frangos de corte, resultado de uma demanda mundial crescente por proteína animal, ovos de matrizes novas têm sido incubados, acarretando maior variação no peso inicial de pintinhos de 1 dia (Oliveira, 1981; Okada, 1994; Castro, 1996).

Pintos com diferentes pesos corporais ao alojamento apresentam curvas de crescimento diferenciadas, o que pode resultar em diferenças de desempenho, normalmente favoráveis às aves com maior peso corporal inicial, pois sabe-se que o peso à eclosão ou peso no momento do alojamento é altamente correlacionado ao peso vivo durante o crescimento de frangos de corte, portanto, é de grande interesse para a indústria avícola (Maiorka et al., 2003).

Objetivou-se com este trabalho avaliar os efeitos do tempo de fornecimento da dieta pré-inicial a pintinhos com diferentes pesos ao alojamento sobre o desempenho produtivo e o rendimento de carcaça de frangos de corte até os 49 dias de idade.

\section{Material e Métodos}

O experimento foi conduzido no aviário experimental da Faculdade de Medicina Veterinária e Zootecnia - USP, em Pirassununga, São Paulo, durante o período de janeiro a março de 2006.

Foram utilizados 960 pintinhos de corte machos, linhagem comercial Cobb ${ }^{\circledR}$, provenientes do mesmo lote de matrizes pesadas (48 semanas de idade), fornecidas por um incubatório local. As aves foram distribuídas em delineamento inteiramente casualizado em esquema fatorial $2 \times 4$, composto de 2 pesos (42 e 46 g) e 4 períodos de fornecimento da dieta pré-inicial (0, 1 a 7, 1 a 10 e 1 a 14 dias), totalizando oito tratamentos, cada um com quatro repetições de 30 aves. As aves foram criadas durante 49 dias com água e alimentação à vontade. Os esquemas de alimentação adotados consistiram de três (para aves não alimentadas com a DPI) ou quatro fases (para aves que receberam a DPI). As dietas experimentais (Tabela 1 ) foram formuladas à base de milho e farelo de soja, conforme níveis estabelecidos por Rostagno et al. (2005).

Utilizou-se um galpão convencional de alvenaria, com cumeeira tendo orientação leste-oeste, coberto com telhas de barro, com mureta lateral de alvenaria de $0,40 \mathrm{~m}$, protegido por cortina de plástico azul, com sistema móvel de catraca para sua movimentação no controle do conforto térmico. Os boxes $\left(4,25 \mathrm{~m}^{2}\right)$ foram forrados com cama de maravalha e equipados durante as fases iniciais com lâmpada infravermelha, para aquecimento dos pintos, comedouros tipo tubular infantil e bebedouros de pressão. O controle do aquecimento, bem como o manejo das cortinas, foi realizado de acordo com a necessidade das aves; a aferição das condições ambientais foi realizada com o auxílio de dois data loggers da marca Hobo ${ }^{\circledR}$ (Tabela 2). Gradativamente, os equipamentos para a fase inicial foram substituídos por

Tabela 1 - Composição e valores nutricionais das dietas experimentais

\begin{tabular}{|c|c|c|c|c|}
\hline \multirow[t]{2}{*}{ Ingrediente } & \multicolumn{4}{|c|}{ Dieta e período de fornecimento ${ }^{1}$} \\
\hline & $\begin{array}{l}\text { Pré-inicial } \\
\text { Variável }\end{array}$ & $\begin{array}{c}\text { Inicial } \\
\text { até } 21 \\
\text { dias }\end{array}$ & $\begin{array}{c}\text { Engorda } \\
22-42 \\
\text { dias }\end{array}$ & $\begin{array}{c}\text { Final } \\
42-49 \\
\text { dias }\end{array}$ \\
\hline Fubá de milho & 53,23 & 54,47 & 59,27 & 63,12 \\
\hline Farelo de soja & 38,70 & 36,80 & 31,20 & 27,00 \\
\hline Calcário calcítico & 0,80 & 0,90 & 1,00 & 1,10 \\
\hline Fosfato bicálcico & 2,00 & 1,90 & 1,70 & 1,50 \\
\hline Sal & 0,31 & 0,41 & 0,35 & 0,25 \\
\hline Óleo de soja & 3,40 & 4,50 & 5,40 & 5,70 \\
\hline L-lisina $(50,7 \%)$ & 0,55 & 0,27 & 0,31 & 0,39 \\
\hline DL-metionina (99\%) & 0,16 & 0,05 & 0,05 & 0,11 \\
\hline Bicarbonato de sódio & 0,35 & 0,20 & 0,22 & 0,33 \\
\hline $\begin{array}{l}\text { Suplemento vitamínico- } \\
\text { mineral }^{2}\end{array}$ & 0,50 & 0,50 & 0,50 & 0,50 \\
\hline \multicolumn{5}{|l|}{ Composição calculada } \\
\hline $\begin{array}{l}\text { Energia metabolizável } \\
(\mathrm{kcal} / \mathrm{kg})\end{array}$ & 2.961 & 3.051 & 3.181 & 5.24 \\
\hline Proteína bruta (\%) & 22,11 & 21,14 & 19,00 & 17,50 \\
\hline Arginina digestível (\%) & 1,34 & 1,29 & 1,14 & 1,03 \\
\hline Isoleucina digestível (\%) & 0,89 & 0,86 & 0,76 & 0,69 \\
\hline Lisina digestível (\%) & 1,36 & 1,19 & 1,07 & 1,02 \\
\hline Metionina digestível (\%) & 0,65 & 0,53 & 0,49 & 0,46 \\
\hline $\begin{array}{l}\text { Metionina+cistina } \\
\text { digestível (\%) }\end{array}$ & 0,97 & 0,84 & 0,77 & 0,73 \\
\hline Treonina digestível (\%) & 0,73 & 0,71 & 0,63 & 0,58 \\
\hline Triptofano digestível (\%) & 0,25 & 0,24 & 0,21 & 0,19 \\
\hline Fósforo disponível (\%) & 0,47 & 0,45 & 0,40 & 0,37 \\
\hline Cálcio (\%) & 0,95 & 0,95 & 0,90 & 0,90 \\
\hline Colina (ppm) & 1.516 & 1.482 & 1.280 & 1.095 \\
\hline Sódio (\%) & 0,22 & 0,21 & 0,20 & 0,19 \\
\hline
\end{tabular}

${ }^{1}$ Valores expressos em porcentagem.

2 Fornecendo por kg das dietas: pré-inicial e inicial = ácido fólico - 0,43 mg ácido pantotênico - 9,95 mg; antioxidante - 1,00 mg; biotina - 85,00 mcg $\mathrm{Cu}$ - 8,00 mg; coccidiostático - 50,00 mg; colina - 475,00 mg; Fe - 50,00 mg; I - 0,75 mg; Mn - 75,00 mg; menadiona - 2,10 mg; metionina - 1,83 mg; niacina - 31,35 mg; piridoxina - 0,85 mg; promotor de crescimento - 17,50 mg; riboflavina - 6,65 mg; Se - 0,30 mg; tiamina - 1,45 mg; vit. A - 8.350,00 UI; vit. B12 - 15,00 mcg; vit. D3 - 2.100,00 UI; vit. E - 16,70 UI; Zn - 50,00 mg; Engorda = ácido fólico - 0,80 mg; ácido pantotênico - 10,00 mg; biotina 40,00 mcg; Cu - 122,50 mg; colina - 370,00 mg; Fe - 60,00 mg; I - 1,00 mg $\mathrm{Mn}$ - 70,00 mg; metionina - 1.500,00 mg; niacina - 30,00 mg; piridoxina $2,40 \mathrm{mg}$; riboflavina - 5,00 mg; Se - 0,30 mg; tiamina - 1,50 mg; vit. A 7.000,00 UI; vit. B12 - 10,00 mcg; vit. D3 - 1.600,00 UI; vit. E - 14,00 UI; vit. K3 - 1,80 mg; Zn - 50,00 mg; Final = ácido fólico - 0,18 mg; ácido pantotênico $4,30 \mathrm{mg}$; antifúngico - 25,00 mg; antioxidante - $1,00 \mathrm{mg}$; biotina - $35,00 \mathrm{mcg}$ $\mathrm{Cu}$ - 8,00 mg; colina - 256,00 mg; Fe - 50,00 mg; I - 0,75 mg; Mn - 75,00 mg menadiona - 0,90 mg; metionina - 1,08 mg; niacina - 13,50 mg; piridoxina $0,36 \mathrm{mg}$; promotor de crescimento - $17,50 \mathrm{mg}$; riboflavina - 2,90 mg; Se $0,20 \mathrm{mg}$; tiamina - 0,65 mg; vit. A - 3.600,00 UI; vit. B12 - 6,50 mcg; vit D3 - 900,00 UI; vit. E - 7,20 UI; Zn - 50,00 mg. 
Tabela 2 - Temperaturas máxima, mínima e média, registradas dentro do galpão experimental durante o período de realização do experimento ${ }^{1}$

\begin{tabular}{cccc}
\hline Idade (semanas) & Máxima & Mínima & Média \\
\hline 1 & $32,2 \pm 1,2$ & $21,8 \pm 1,0$ & $27,0 \pm 0,8$ \\
2 & $31,9 \pm 1,6$ & $23,2 \pm 1,3$ & $27,5 \pm 0,7$ \\
3 & $31,6 \pm 2,4$ & $22,0 \pm 1,2$ & $26,9 \pm 1,6$ \\
4 & $33,0 \pm 2,5$ & $23,2 \pm 2,1$ & $28,1 \pm 2,2$ \\
5 & $27,9 \pm 3,0$ & $22,6 \pm 1,3$ & $25,2 \pm 1,8$ \\
6 & $28,7 \pm 2,6$ & $21,6 \pm 1,7$ & $25,1 \pm 1,4$ \\
7 & $28,7 \pm 2,2$ & $21,8 \pm 2,0$ & $25,2 \pm 1,5$ \\
Média & $30,5 \pm 2,9$ & $22,3 \pm 1,7$ & $26,4 \pm 1,8$ \\
\hline
\end{tabular}

${ }^{1}$ Valores expressos em ${ }^{\circ} \mathrm{C}$ como média \pm desvio-padrão para cada semana.

comedouros tubulares e bebedouros pendulares, e o aquecimento deixou de ser utilizado. O programa de luz adotado compreendeu 24 horas de iluminação em todas as fases de criação. As aves foram vacinadas contra newcastle e gumboro via ocular, no $10^{\circ}$ dia de idade.

Foram avaliados, como medidas de desempenho, o ganho de peso, o consumo de ração e a conversão alimentar. Ao final do experimento, após 6 horas de jejum, três aves de cada repetição foram selecionadas de acordo com o peso médio de cada unidade experimental e abatidas para avaliação da carcaça, quando se considerou o peso da carcaça eviscerada em relação ao peso vivo da ave após o jejum. Foram mensurados o peso relativo de vísceras comestíveis, e da gordura abdominal em relação ao peso vivo das aves. Definiu-se como gordura abdominal o tecido adiposo presente em volta da cloaca, bursa de Fabricius e dos músculos abdominais adjacentes, conforme descrito por Smith (1993). Determinaram-se também a relação percentual do peso do peito com osso (sem pele), pernas (coxa e sobre coxa), asas e dorso em relação ao peso da carcaça.

O abate foi realizado no abatedouro da Faculdade de Zootecnia e Engenharia de Alimentos - USP, do campus de Pirassununga, São Paulo. Os procedimentos de abate e corte das carcaças foi realizado por pessoas treinadas, e de maneira similar a de um abatedouro comercial de frangos de corte.

Os dados obtidos foram analisados com auxílio do procedimento GLM (SAS, 2003) e as médias comparadas pelo teste Tukey a $5 \%$ de probabilidade.

\section{Resultados e Discussão}

Não foi verificado efeito de interação significativa $(\mathrm{P}>0,05)$ para nenhum dos fatores estudados. O peso dos pintinhos ao alojamento afetou significativamente o consumo de ração, até os 42 dias de idade, e o ganho de peso das aves (Tabelas 3, 4 e 5) durante todo o período experi- mental $(\mathrm{P}<0,05)$. Não foram observadas diferenças significativas para a conversão alimentar das aves $(\mathrm{P}>0,05)$. De modo geral, pintinhos mais pesados ao alojamento apresentaram melhor desempenho durante o período total do experimento, com peso vivo aos 49 dias de idade 1,8\% superior ao dos pintinhos alojados com menor peso. Assim, cada grama a mais ao alojamento representou $15,5 \mathrm{~g}$ a mais ao abate. Os resultados observados estão de acordo com aqueles relatados por Vieira \& Moran (1998a,b), Araújo et al. (1999), Sklan et al. (2003), Stringhini et al. (2003). Segundo Vieira (2001), o peso vivo de frangos de corte ao abate está positivamente correlacionado ao peso do ovo incubável, o que está correlacionado ao peso do pintinho à eclosão. Pinchasov (1991) postulou que o desempenho superior ao das aves mais pesadas à eclosão pode estar relacionado ao maior consumo de ração.

As aves alimentadas com dieta pré-inicial durante14 dias apresentaram ganho de peso e conversão alimentar inferiores $(\mathrm{P}<0,05)$ nos primeiros 21 dias de idade e, conseqüentemente, pior desempenho geral, o que pode estar relacionado ao excesso de nutrientes da dieta. De acordo com Araújo et al. (2002), o excesso de aminoácidos na dieta causa sobrecarga nos sistemas hepático e renal dos animais, afetando negativamente a produção, em virtude do dispêndio de energia necessário para metabolizar e excretar esses compostos.

Foram observados efeitos significativos no desempenho das aves quando o período total do experimento foi considerado. Aves alimentadas com a dieta pré-inicial por um período de sete dias apresentaram maior $(\mathrm{P}<0,05)$ consumo de ração e ganho de peso em comparação àquelas que não receberam a dieta pré-inicial. Ao término do experimento, as aves alimentadas com dieta pré-inicial durante sete dias tiveram peso vivo $3 \%$ superior ao das aves alimentadas apenas com a dieta inicial.

Os resultados observados neste experimento estão de acordo com aqueles relatados por Silva et al. (2004), entretanto, diferem daqueles observados por Franco et al. (1983), Zúniga et al. (1994), Shiroma et al. (1996), Araújo et al. (1999) e Stringhini et al. (2003). Segundo Lilburn (1998), a dieta préinicial deve ser utilizada nos primeiros sete ou dez dias de vida das aves e ser considerada um investimento, e não um custo no sistema de produção. Outros autores afirmam ainda que a dieta pré-inicial poderia ser adotada por um período de 7 até 14 dias (Franco et al., 1983; Zúniga et al., 1994; Shiroma et al., 1996; Stringhini et al., 2003; Silva et al., 2004). Assim, o nutricionista deve se atentar à utilização conjunta ao milho de ingredientes de alta digestibilidade e de elevado teor protéico, atendendo às exigências nutricionais da ave neonata. 
Tabela 3 - Consumo de ração de frangos de corte alimentados com dieta pré-inicial por vários períodos e com diferentes pesos ao alojamento no período de 1 a 49 dias de idade

\begin{tabular}{|c|c|c|c|c|c|}
\hline & \multicolumn{5}{|c|}{ Idade (dias) } \\
\hline & 1 a 7 & 1 a 14 & 1 а 21 & 1 a 42 & 1 а 49 \\
\hline & \multicolumn{5}{|c|}{ Período de fornecimento da dieta pré-inicial (dias) } \\
\hline 0 & $147 \pm 4 b$ & $558 \pm 12 \mathrm{a}$ & $1.395 \pm 24$ & $4.988 \pm 64$ & $6.376 \pm 88 b$ \\
\hline 1 a 7 & $154 \pm 3 a$ & $579 \pm 13 a$ & $1.392 \pm 25$ & $5.073 \pm 92$ & $6.561 \pm 154 a$ \\
\hline 1 a 10 & $152 \pm 3 a$ & $576 \pm 11 a$ & $1.369 \pm 29$ & $5.047 \pm 101$ & $6.504 \pm 147 \mathrm{ab}$ \\
\hline \multirow[t]{2}{*}{1 a 14} & $152 \pm 5 a$ & $588 \pm 21 b$ & $1.375 \pm 24$ & $5.081 \pm 105$ & $6.562 \pm 139 a$ \\
\hline & \multicolumn{5}{|c|}{ Peso inicial do pintinho } \\
\hline Pesado & $154 \pm 4 a$ & $583 \pm 18 a$ & $1.393 \pm 20 \mathrm{a}$ & $5.094 \pm 80 a$ & $6.537 \pm 169$ \\
\hline Leve & $149 \pm 3 b$ & $568 \pm 14 b$ & $1.372 \pm 29 b$ & $5.001 \pm 87 b$ & $6.465 \pm 122$ \\
\hline Interação ${ }^{1}$ & NS & NS & NS & NS & NS \\
\hline
\end{tabular}

IInteração período de fornecimento da dieta pré-inicial x peso inicial do pintinho; Valores expressos em $\mathrm{kg} / \mathrm{kg}$.

Médias ( \pm desvio-padrão) sobrescritas com letras distintas na coluna para a mesma variável diferem $(P<0,05)$ pelo teste Tukey; NS - não-significativo.

Tabela 4 - Ganho de peso de frangos de corte alimentados com dieta pré-inicial durante vários períodos e com diversos pesos ao alojamento no período de 1 a 49 dias de idade

\begin{tabular}{|c|c|c|c|c|c|}
\hline & \multicolumn{5}{|c|}{ Idade (dias) } \\
\hline & 1 a 7 & 1 a 14 & 1 a 21 & 1 a 42 & 1 a 49 \\
\hline & \multicolumn{5}{|c|}{ Período de fornecimento da dieta pré-inicial (dias) } \\
\hline 0 & $150 \pm 4 b$ & $498 \pm 10 a$ & $1019 \pm 20 a$ & $2953 \pm 51$ & $3457 \pm 50 b$ \\
\hline 1 a 7 & $152 \pm 3 a b$ & $497 \pm 8 a$ & $1021 \pm 22 a$ & $3012 \pm 50$ & $3563 \pm 92 \mathrm{a}$ \\
\hline 1 a 10 & $153 \pm 2 \mathrm{a}$ & $493 \pm 10 \mathrm{a}$ & $1007 \pm 20 \mathrm{a}$ & $2982 \pm 92$ & $3489 \pm 103 a b$ \\
\hline \multirow[t]{2}{*}{1 a 14} & $152 \pm 4 \mathrm{ab}$ & $484 \pm 9 b$ & $981 \pm 25 b$ & $3000 \pm 75$ & $3554 \pm 55 a b$ \\
\hline & \multicolumn{5}{|c|}{ Peso inicial do pintinho } \\
\hline Pesado & $154 \pm 3 a$ & $500 \pm 7 a$ & $1021 \pm 24 a$ & $3015 \pm 64 a$ & $3545 \pm 82 a$ \\
\hline Leve & $149 \pm 3 b$ & $486 \pm 7 b$ & $994 \pm 22 b$ & $2958 \pm 64 b$ & $3487 \pm 85 b$ \\
\hline Int. $\mathrm{D} \times \mathrm{P}^{1}$ & NS & NS & NS & NS & NS \\
\hline
\end{tabular}

Tabela 5 - Conversão alimentar de frangos de corte alimentados com dieta pré-inicial durante vários períodos e com diferentes pesos ao alojamento no período de 1 a 49 dias de idade

\begin{tabular}{|c|c|c|c|c|c|}
\hline & \multicolumn{5}{|c|}{ Idade (dias) } \\
\hline & \multicolumn{5}{|c|}{ Período de fornecimento da dieta pré-inicial (dias) } \\
\hline 0 & $0,99 \pm 0,01 \mathrm{a}$ & $1,12 \pm 0,01 \mathrm{a}$ & $1,37 \pm 0,03 \mathrm{ab}$ & $1,69 \pm 0,03$ & $1,84 \pm 0,03$ \\
\hline 1 a 7 & $1,01 \pm 0,02 b$ & $1,16 \pm 0,02 b$ & $1,36 \pm 0,03 \mathrm{ab}$ & $1,68 \pm 0,01$ & $1,84 \pm 0,03$ \\
\hline 1 a 10 & $0,99 \pm 0,01 \mathrm{ab}$ & $1,17 \pm 0,03 b$ & $1,36 \pm 0,04 a$ & $1,69 \pm 0,03$ & $1,87 \pm 0,04$ \\
\hline Pesado & $1,00 \pm 0,02$ & $1,16 \pm 0,05$ & $1,36 \pm 0,03$ & $1,69 \pm 0,03$ & $1,84 \pm 0,03$ \\
\hline Leve & $1,00 \pm 0,02$ & $1,17 \pm 0,04$ & $1,38 \pm 0,03$ & $1,69 \pm 0,03$ & $1,85 \pm 0,03$ \\
\hline Int. $\mathrm{D} \times \mathrm{P}^{1}$ & NS & NS & NS & NS & NS \\
\hline
\end{tabular}

${ }^{1}$ Interação de período de fornecimento da dieta pré-inicial e peso inicial do pintinho; Valores expressos em $\mathrm{kg} / \mathrm{kg}$.

Médias ( \pm desvio-padrão) sobrescritas com letras distintas na coluna, e para a mesma variável, diferem $(P<0,05)$ pelo teste Tukey; NS - não-significativo. 
Tabela 6 - Rendimento de carcaça, cortes, vísceras comestíveis e gordura abdominal de frangos de corte alimentados com dieta préinicial por vários períodos e com diversos pesos ao alojamento no período de 1 a 49 dias de idade ${ }^{1,2}$

\begin{tabular}{|c|c|c|c|c|c|c|c|c|c|}
\hline & Coração & Moela & Fígado & Gordura & Carcaça & Pernas & Asas & Peito & Dorso \\
\hline \multicolumn{10}{|c|}{ Período de fornecimento da dieta pré-inicial (dias) } \\
\hline 0 & $0,4 \pm 0,1$ & $1,4 \pm 0,3$ & $1,3 \pm 0,2 b$ & $1,4 \pm 0,4$ & $73,4 \pm 1,5 a b$ & $31,4 \pm 1,7$ & $11,0 \pm 0,6$ & $37,8 \pm 2,3$ & $20,2 \pm 1,1$ \\
\hline 1 a 7 & $0,4 \pm 0,0$ & $1,3 \pm 0,2$ & $1,5 \pm 0,2 \mathrm{a}$ & $1,6 \pm 0,5$ & $73,1 \pm 1,1 b$ & $31,1 \pm 1,6$ & $11,0 \pm 0,5$ & $37,9 \pm 1,6$ & $20,0 \pm 0,8$ \\
\hline 1 a 10 & $0,4 \pm 0,1$ & $1,4 \pm 0,3$ & $1,4 \pm 0,2 \mathrm{ab}$ & $1,6 \pm 0,6$ & $73,3 \pm 1,6 a b$ & $31,8 \pm 1,4$ & $10,8 \pm 0,4$ & $38,0 \pm 1,6$ & $19,7 \pm 0,9$ \\
\hline 1 a 14 & $0,4 \pm 0,0$ & $1,4 \pm 0,3$ & $1,4 \pm 0,2 b$ & $1,4 \pm 0,4$ & $74,2 \pm 1,1 \mathrm{a}$ & $30,6 \pm 1,2$ & $10,8 \pm 0,5$ & $38,3 \pm 1,8$ & $20,2 \pm 1,3$ \\
\hline \multicolumn{10}{|c|}{ Peso inicial do pintinho } \\
\hline Pesado & $0,4 \pm 0,1$ & $1,3 \pm 0,3$ & $1,4 \pm 0,2$ & $1,6 \pm 0,5$ & $73,6 \pm 1,2$ & $31,3 \pm 1,3$ & $10,8 \pm 0,5$ & $38,0 \pm 1,5$ & $19,9 \pm 0,9$ \\
\hline Leve & $0,4 \pm 0,1$ & $1,4 \pm 0,3$ & $1,4 \pm 0,2$ & $1,5 \pm 0,4$ & $73,4 \pm 1,5$ & $31,2 \pm 1,7$ & $11,0 \pm 0,5$ & $38,0 \pm 2,1$ & $20,2 \pm 1,1$ \\
\hline Int. $\mathrm{D} \times \mathrm{P}^{3}$ & NS & NS & NS & NS & NS & NS & NS & NS & NS \\
\hline
\end{tabular}

1 Peso relativo de coração, moela, fígado, gordura abdominal e da carcaça eviscerada em relação ao peso vivo.

2 Percentual de pernas (coxa+sobrecoxa), asas, peito (com osso e sem pele) e dorso em relação à carcaça eviscerada.

3 Interação período de fornecimento da dieta pré-inicial $x$ peso inicial do pintinho.

Médias ( \pm desvio-padrão) sobrescritas com letras distintas na coluna para a mesma variável diferem pelo teste Tukey (P<0,05); NS - não-significativo.

O peso dos pintinhos ao alojamento não afetou $(\mathrm{P}>0,05)$ nenhuma das características de carcaça analisadas (Tabela 6). Em estudo conduzido por Joseph \& Moran Jr. (2005b), houve diferença significativa na quantidade de gordura abdominal de frangos de corte de diferentes pesos ao alojamento, uma vez que pintinhos mais pesados apresentaram maior percentual de gordura. Entretanto, os pintinhos eram provenientes de matrizes de diferentes idades, os pintinhos pesados eram provenientes de matrizes com 41semanas de idade e os leves, de matrizes de 32 semanas de idade.

Neste experimento, foram observadas diferenças significativas $(\mathrm{P}<0,05)$ nas características de carcaça quando a dieta pré-inicial foi administrada por diferentes períodos. São raros os trabalhos na literatura para avaliação dos efeitos dos períodos de fornecimento da dieta e do peso ao alojamento sobre as características de carcaça.

Foi observado efeito $(\mathrm{P}<0,05)$ do período de fornecimento da dieta pré-inicial no peso relativo de fígado de aves abatidas com 49 dias de idade. Aves alimentadas com dieta pré-inicial por sete dias apresentaram maior percentual de fígado ao abate em comparação àquelas que receberam a dieta pré-inicial por 14 dias, ou àquelas que não receberam a dieta. Esse resultado pode explicar, em parte, o melhor desempenho das aves que receberam a dieta pré-inicial por sete dias, uma vez que o fígado está envolvido em uma série de processos metabólicos e fisiológicos do organismo. Ressalta-se que todos os fígados analisados não apresentavam sinais de lesão nem acúmulo de gordura e aparentavam-se todos íntegros. Os resultados encontrados neste estudo divergem daqueles observados por Stringhini et al. (2003) e Silva et al. (2004).

Observou-se ainda efeito significativo para rendimento de carcaça de frangos alimentados com dieta préinicial por diversos períodos. Aves alimentadas com a dieta pré-inicial por 14 dias apresentaram maior percentual de rendimento de carcaça em comparação àquelas alimentadas por sete dias com a dieta pré-inicial. Em estudo conduzido por Rodrigueiro et al. (2000), não foram observados efeitos significativos do período de fornecimento da dieta pré-inicial sobre o rendimento de carcaça e o percentual relativo de gordura abdominal.

\section{Conclusões}

O peso inicial de pintinhos de corte ao alojamento não influencia as características de carcaça, mas é fator determinante no desempenho das aves, que é melhor nas aves mais pesadas. O fornecimento da dieta pré-inicial durante sete dias favorece o desempenho durante todo o período experimental, mas diminui o rendimento de carcaça das aves.

\section{Literatura Citada}

ARAÚJO, C.S.S.; STRINGHINI, J.H.; ARAÚJO, L.F. et al. Manejo nutricional de frangos de corte na fase pré-inicial. Archivos Latino-americanos de Producción Animal, v.7, p.77-84, 1999.

ARAÚJO, L.F.; JUNQUEIRA, O.M.; ARAÚJO, C.S.S. et al. Different criterion of feed formulation for broilers in the period 1 to 21 days of age. Brazilian Journal of Poultry Science, v.4, p.195-202, 2002.

CASTRO, A.G.M. Qualidade de pintos de um dia e importância do manejo no desempenho de frangos de corte. In: SIMPÓSIO GOIANO DE AVICUlTuRA, 1996, Goiânia. Anais... Goiânia: Associção Goiana de Avicultura, 1996. p.67-70.

CROOM, W.J.; BRAKE, J.; COLES, B.A. et al. Is intestinal absorption capacity rate-limiting for performance in poultry? Journal of Applied Poultry Research, v.8, p.242-252, 1999.

FRANCO, S.G.; CURVELLO, F.A.; CAMPOS, E.J. et al. Programas alimentares para frangos de corte: I. Efeitos sobre o desempenho. Arquivos Brasileiros de Medicina Veterinária e Zootecnia, v.3, p.405-415, 1983. 
JOSEPH, N.S.; MORAN JR., E.T. Characteristics of eggs, embryos, and chicks from broiler breeder hens selected for growth or meat yield. Journal of Applied Poultry Research, v.14, p.275-280, 2005a.

JOSEPH, N.S.; MORAN JR., E.T. Effect of flock age and postemergent holding in the hatcher on broiler live performance and further-processing yield. Journal of Applied Poultry Research, v.14, p.512-520, 2005b.

LILBURN, M.S. Practical aspects of early nutrition for poultry. Journal of Applied Poultry Research, v.7, p.420-424, 1998.

MAIORKA, A.; LUQUETTI, B.C.; ALMEIDA, J.G. et al. Idade da matriz e qualidade do pintinho. In: MACARI, M.; GONZALES, E. (Eds.). Manejo da incubação. 2.ed. Jaboticabal: Fundação Apinco de Ciência e Tecnologia Avícolas, 2003. p.361-377.

MORAN JR., E.T. Digestion and absorption of carbohydrates in fowl and events through perinatal development. Journal of Nutrition, v.115, p.665-671, 1985.

OKADA, T.M.A. Qualidade do pinto de um dia: In: CONFERÊNCIA APINCO DE CIÊNCIA E TECNOLOGIA AVÍCOLAS, 1994, Campinas. Anais... Campinas: Fundação Apinco de Ciência e Tecnologia Avícolas, 1994. p.41-46.

OLIVEIRA, R.L. Mortalidade inicial - causas e controle. In: CAMPOS, E.J.; LAMAS DA SILVA, J.M.; SILVA, E.N. (Eds.) Produção e qualidade de pintos de um dia. Belo Horizonte: 1981. p.229-236.

PINCHASOV, Y. Relationship between the weight of hatching eggs and subsequent early performance of broiler chicks. British Poultry Science, v.32, p.109-115, 1991.

RODRIGUEIRO, R.J.B.; MURAKAMI, A.E.; POZZA, P.C. et al. Efeito de dois programas de alimentação sobre o desempenho e o rendimento de carcaça de duas marcas comerciais de frangos de corte. Revista Brasileira de Zootecnia, v.29, p.502-506, 2000 .

ROSTAGNO, H.S.; ALBINO, L.F.T.; DONZELE, J.L. Tabelas brasileiras para aves e suínos: composição de alimentos e exigências nutricionais. Viçosa, MG: Editora UFV, 2005. 186p.
STATISTICAL ANALYSIS SYSTEM - SAS. SAS system for Microsoft Windows version 8.2. Cary: Statistical Analysis Systems, 2003. (CD-ROM).

SHIROMA, N.N.; ZANETTI, M.A.; FARIA, D.E. et al. Efeito de programas alimentares sobre o desempenho de frangos de corte durante o inverno. In: CONFERÊNCIA APINCO DE CIÊNCIA E TECNOLOGIAS AVÍCOLAS. Anais... Campinas: Fundação Apinco de Ciência e Tecnologia Avícolas, 1996. p.51.

SILVA, J.R.L.; RABELLO, C.B.; DUTRA JR., W.M.D. et al. Efeito da forma física e do programa alimentar na fase pré-inicial sobre o desempenho e características de carcaça de frangos de corte. Acta Scientiarum, v.26, p.543-551, 2004.

SKLAN, D.; HEIFETZ, S.; HALEVY, O. Heavier chicks at hatch improves marketing body weight by enhancing skeletal muscle growth. Poultry Science, v.82, p.1778-1786, 2003.

SMITH, M.O. Parts yield of broilers reared under cycling high temperatures. Poultry Science, v.72, p.1146-1150, 1993.

STRINGHINI, J.H.; RESENDE, A.; CAFÉ, M.B. et al. Efeito do peso inicial dos pintos e do período da dieta pré-inicial sobre o desempenho de frangos de corte. Revista Brasileira de Zootecnia, v.32, p.353-360, 2003.

VIEIRA, S.L. Idade da matriz, tamanho do ovo e desempenho do pintinho. In: CONFERÊNCIA APINCO DE CIÊNCIA E TECNOLOGIA AVÍCOlAS, 2001, Campinas. Anais... Campinas: Fundação Apinco de Ciência e Tecnologia Avícolas, 2001. p.117-123.

VIEIRA, S.L.; MORAN JR., E.T. Broiler yields using chicks from egg weight extremes in breeder age and dietary propionate. Journal of Applied Poultry Research, v.7, p.320-327, 1998a.

VIEIRA, S.L.; MORAN JR., E.T. Broiler yields using chicks from egg weight extremes and diverse strains. Journal of Applied Poultry Research, v.7, p.339-346, 1998b.

ZÚNIGA, I.O.; CAMPOS, E.J.; FERREIRA, J.M. et al. Efeito de programas de alimentação sobre o desempenho de frangos de corte. Arquivos Brasileiros de Medicina Veterinária e Zootecnia, v.46, p.675-683, 1994. 